\title{
Is Alzheimer's a preventable disease? Roger Pinder
}

\author{
Address: Institute of Psychiatry, London, UK \\ from International Society on Brain and Behaviour: 3rd International Congress on Brain and Behaviour \\ Thessaloniki, Greece. 28 November - 2 December 2007 \\ Published: 17 April 2008 \\ Annals of General Psychiatry 2008, 7(Suppl I):S59 doi:I0.II86/I744-859X-7-SI-S59
}

This abstract is available from: http://www.annals-general-psychiatry.com/content/7/SI/S59

(C) 2008 Pinder; licensee BioMed Central Ltd.

The incidence of Alzheimer' disease (AD) in the developed countries increases with age, from about $1 \%$ in those aged 60-64 years to over $45 \%$ for those aged 95 years and over. With demographic ageing occurring rapidly in China, India and Latin America, it is likely that AD will become a major medical concern also in the developing countries. Some symptomatic treatments are available in the form of the cholinesterase inhibitors donepezil, galantamine and rivastigmine, as well as the NMDA receptor antagonist memantine. Current therapeutic targets include the enzymes involved in the formation or degradation of the amyloid or tau proteins responsible for the classical Alzheimer pathology of plaques and tangles. Therapeutic vaccines are also being developed to target aberrant proteins as soon as they appear in the ageing brain. AD may well be preventable in the future.

There is also substantial evidence that diet may play an important role in lowering the risk for developing AD. For example, there is a lower incidence of $\mathrm{AD}$ in societies which consume a Mediterranean diet of mainly fish, fruit, vegetables, olive oil and wine. In particular, there is widespread evidence from both population-based cohort and case control studies that regular consumption of moderate amounts of alcohol in the form of wine reduces the risks of developing $\mathrm{AD}$ compared with abstainers and heavy drinkers. It also appears that age-related cognitive decline, particularly in women, is hampered in regular drinkers, and that older drinkers with diagnosable Mild Cognitive Impairment (MCI) progress less frequently to AD than their abstaining counterparts. Plausible biological mechanism exist for the protective effects of wine, including antioxidant and inflammatory properties of the polyphenols present in wine, particularly in red varietals, which are extracted during fermentation. 